\title{
Environment-induced corrections to the spin Hamiltonian as dynamic frequency shifts in nuclear magnetic resonance
}

\author{
G. Karthik ${ }^{\text {a) }}$ \\ Department of Physics, Indian Institute of Science, Bangalore, India \\ Anil Kumar') \\ Department of Physics and Sophisticated Instruments Facility, Indian Institute of Science, Bangalore, India
}

\begin{abstract}
We derive an expression for the correction to the spin-system Hamiltonian that arises due to the system-bath interaction, starting both from the standard master equation for the spin density matrix and a perturbative diagonalization of the system-bath Hamiltonian to the second order in the interaction. We show that the dynamic frequency shifts observed in the evolution of the nuclear spin coherences are a result of these Hamiltonian corrections. We present a systematic decomposition of the relaxation superoperator into Hermitian and anti-Hermitian parts as opposed to the usual practice of partitioning it into real and imaginary parts. We point out that the relaxation-induced corrections to the coherent motion arise exclusively from the anti-Hermitian part and the dissipative effects, from the Hermitian part, both, in general, being complex. However, the secular terms of this correction are found to depend only on the imaginary and the real parts, respectively.
\end{abstract}

\section{INTRODUCTION}

The relaxation behavior of a system of nuclear spins in contact with the other molecular degrees of freedom is captured by a master equation for the spin density matrix. ${ }^{1-3}$ The Redfield equation is one such master equation that is used to describe all relaxation phenomena in the NMR of liquids. The coupling of the spin system to the bath comprised of the molecular motional degrees of freedom changes the nature of the evolution of the spin system in a fundamental way, in that it introduces irreversibility. In this description, the bath is modeled as a classical object undergoing random motion characterized by the spectral density function. ${ }^{4}$ The random interactions give rise to both dissipative and coherent corrections to the motion of the system. These coherent effects induced by the random interactions manifest themselves in the form of shifts in the frequencies known as dynamic frequency shifts ${ }^{5-13}$ (henceforth referred to as DFS), and are the subject of this article.

In contrast to the line broadenings and the recovery of longitudinal magnetization produced by the dissipative part of the relaxation superoperator, these shifts are very difficult to observe due to the absence of any reference scale against which these could be measured. These shifts have been observed when they, arising from the cross-correlation between different relaxation mechanisms, either destroy the symmetry of a multiplet ${ }^{5,6}$ or produce field-dependent changes in the coupling values. ${ }^{9}$

There has been a considerable amount of work, both theoretical and experimental, on the manifestations of these shifts in various coupled spin systems. It is often stated that

\footnotetext{
a)Electronic mail: kart@sif.iisc.ernet.in

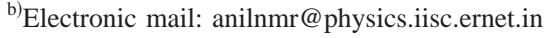

these shifts arise out of the imaginary part of the spectral densities while the real part only contributes to purely dissipative evolution. Recently, Brüschweiler has shown that the DFS in an AX system that arise due to the cross-correlation between the fluctuations of the chemical shift and the dipolar interaction can be represented as a correction to the $\mathrm{J}$ coupling between the spins ${ }^{10}$ and in same spirit, the DFS due to the dipole-dipole cross-correlation in an AMX system is equivalent to a three-spin $\mathrm{J}$ coupling. ${ }^{11}$ The possibility of expressing all DFS as corrections to the Hamiltonian is attractive on account of its notational simplicity and the accompanying simplification in the way one can think about these shifts. More fundamentally, such an equivalence hints at the existence of a refined physical explanation.

In this article, we show that the decomposition of the relaxation superoperator into real and imaginary parts leads to an incomplete picture while a decomposition into Hermitian and anti-Hermitian leads to a more general picture. For example, beyond the secular approximation, ${ }^{14}$ a separation into real and imaginary parts does not correspond to a separation into parts causing, respectively, purely incoherent and purely coherent effects on the evolution while the separation presented in this article is always guaranteed to provide such a correspondence. The Hermitian part contributes only to the purely dissipative evolution while the anti-Hermitian part contributes to purely coherent evolution. An important distinction of the present decomposition is its generality in retaining all the relaxation elements while earlier treatments assumed that the real part of the spectral densities contribute to the decoherence and the imaginary part, to the coherent effects, therby missing out the complex nature of the offdiagonal elements in both the cases. We also present a for- 
malism within the scope of which these shifts naturally arise as corrections to the spins system Hamiltonian.

The layout of the article is as follows. We start from the master equation in Sec. II to show that the relaxation superoperator is decomposable into a Hermitian and an antiHermitian part. While the former accounts for all the irreversibility in the motion the latter produces coherent effects on the system motion. The anti-Hermitian part is shown to have a commutator structure that naturally leads to definition of a correction to the spin Hamiltonian. In Sec. III we start with the system-bath Hamiltonian that is time independent. The system-bath interaction causes the energy levels of the uncoupled system-bath to mix and shift. As a consequence, there is an average shift produced to the energy levels of the system. In order to find these shifts, we use a perturbative diagonalization technique to find the lowest-order corrections to the system levels. This procedure naturally leads to a correction to the system Hamiltonian that we find to be identical to the one derived from the master equation. We then show that the correction contains both diagonal and nondiagonal terms. The diagonal part of the correction is shown to depend only on the imaginary part of the spectral densities. In Sec. IV we provide the Hamiltonian corrections for certain spin systems. In particular, we arrive at the two- and threespin $\mathbf{J}$ couplings in the AX and AMX systems. The crosscorrelation between the quadrupolar and the dipolar interaction in a system of a spin- $\frac{1}{2}-\operatorname{spin}>\frac{1}{2}$ system is found to produce a correction to the system Hamiltonian that destroys the symmetry of the spin- $\frac{1}{2}$ multiplet structure. We also show that the autocorrelation of the quadrupolar interaction for a spin $>1$ system gives rise to a correction to the Hamiltonian that removes the degeneracy of the transitions causing a satellite formation. We conclude the article with Sec. V. In an Appendix we list explicitly, the matrix representation of the complete relaxation superoperator for a two-spins system showing the complex nature of the off-diagonal elements and their consequences.

\section{THE MASTER EQUATION}

In this section, we start with the master equation that describes the evolution of the spin density matrix due to its interaction with a classical bath. The general procedure of obtaining such a master equation in the context of nuclear spin relaxation in liquids is to start with the microscopic evolution equation of the density matrix. The standard second-order treatment in the system-bath interaction and a Markovian approximation leads to the following form for the master equation. 1,2

$$
\frac{d}{d t} \rho=-i \hat{\Gamma}_{s} \rho-\hat{\Gamma} \rho
$$

Here, $\hat{\Gamma}_{s}$ is the Liouvillian of the spin system that generates the free evolution of the system under the spin Hamiltonian and is given in terms of it by the following equation:

$$
\hat{\Gamma}_{s} \rho=\left[\mathcal{H}_{s}, \rho\right] \text {. }
$$

$\hat{\Gamma}$ is the relaxation superoperator that generates the irreversible behavior of the evolution. ${ }^{15}$ In the semiclassical formulation of the master equation, the irreversibility arises due to the rendering of the interaction Hamiltonian $\mathcal{H}^{\prime}$ time dependent due to the random motion of the bath variables. In general, the interaction Hamiltonian can be written as a sum of various scalar contractions of the system and bath variables. We shall use the following notation to represent the general interaction Hamiltonian. ${ }^{15}$

$$
\mathcal{H}^{\prime}=\sum_{\eta, l, q} \chi_{l,-q}^{\eta} \mathcal{A}_{l, q}^{\eta}
$$

Here $\eta$ stands for the various interactions. $l$ and $q$ represent the rank and the azimuthal index of the operators. $\mathcal{A}_{l, q}^{\eta}$ are the operators of the spin system. The isotropy of the bath leads to the following symmetry of the correlation function of the bath variables:

$$
\left\langle\chi_{l,-q}^{\eta}(t) \chi_{l^{\prime}, q^{\prime}}^{\eta^{\prime}}(t+\tau)\right\rangle=\delta_{q, q^{\prime}} \delta_{l, l^{\prime}} \mathcal{C}_{l}^{\eta \eta^{\prime}}(\tau),
$$

where \langle\rangle represents the averaging over the bath variables. Using a second-order perturbation theory and making the Markovian approximation, the following expression for the relaxation superoperator may be derived: ${ }^{3}$

$$
\hat{\Gamma} \rho=\int_{0}^{\infty} d \tau\left\langle\left[\mathcal{H}^{\prime},\left[\tilde{\mathcal{H}}^{\prime}(-\tau), \rho\right]\right]\right\rangle .
$$

The overtilde refers to the operator written in the interaction frame. It is useful to introduce the set of eigenoperators of the free system Liouvillian superoperator,

$$
-i \hat{\Gamma}_{s} \mathcal{S}_{i}=i \omega_{i} \mathcal{S}_{i}
$$

The spin operators that are present in the interaction Hamiltonian can be resolved in this basis to give the following representation:

$$
\mathcal{A}_{l, q}^{\eta}=\sum_{i}\left\langle\mathcal{S}_{i} \mid \mathcal{A}_{l, q}^{\eta}\right\rangle \mathcal{S}_{i}
$$

The coefficients are defined as

$$
\left\langle\mathcal{S}_{i} \mid \mathcal{A}_{l, q}^{\eta}\right\rangle=\operatorname{Tr}\left(\mathcal{S}_{i}^{\dagger} \mathcal{A}_{l, q}^{\eta}\right) \text {. }
$$

Written in terms of these basis operators and using the symmetry property of the bath variables from Eq. (3), we have the following form for $\hat{\Gamma}$ :

$$
\begin{aligned}
\hat{\Gamma} \rho= & \sum_{\eta, \eta^{\prime}, l, q, i, j} \mathcal{J}_{l}^{\eta \eta^{\prime}}\left(\omega_{j}\right)\left\langle\mathcal{S}_{i} \mid \mathcal{A}_{l, q}^{\eta}\right\rangle \\
& \times\left\langle\mathcal{S}_{j} \mid \mathcal{A}_{l,-q}^{\eta^{\prime}}\right\rangle\left[\mathcal{S}_{i},\left[\mathcal{S}_{j}, \rho\right]\right] .
\end{aligned}
$$

We have introduced the spectral density function in the above equation that are defined as follows:

$$
\mathcal{J}_{l}^{\eta \eta^{\prime}}\left(\omega_{j}\right)=\int_{0}^{\infty} d \tau \mathcal{C}_{l}^{\eta \eta^{\prime}}(\tau) \exp i \omega_{j} \tau
$$

The spectral density has the following symmetry that arises out of the reality of the correlation functions: 


$$
\mathcal{J}_{l}^{\eta \eta^{\prime}}(-\omega)=\mathcal{J}_{l}^{\eta \eta^{\prime *}}(\omega)
$$

The complete evolution equation for the density matrix may now be written as

$$
\begin{aligned}
\frac{d}{d t} \rho= & -i \hat{\Gamma}_{s} \rho-\sum_{\eta, \eta^{\prime}, l, q, i, j} \mathcal{J}_{l}^{\eta \eta^{\prime}}\left(\omega_{j}\right)\left\langle\mathcal{S}_{i} \mid \mathcal{A}_{l,(\hat{\sigma}) q}^{\eta^{(\hat{\sigma})}}\right\rangle \\
& \times\left\langle\mathcal{S}_{j} \mid \mathcal{A}_{l,-q}^{\eta^{\prime}}\right\rangle\left[\mathcal{S}_{i},\left[\mathcal{S}_{j}, q\right]\right] .
\end{aligned}
$$

The first term on the RHS corresponds to the free evolution of the system under the spin Hamiltonian. The second term, owing to its double commutator structure, cannot be interpreted as an evolution under a Hamiltonian. It is the source of irreversibility. However, as it stands, the second term is not interpretable as causing pure dissipation since a purely dissipative superoperator has to be Hermitian. It is therefore useful to partition the relaxation superoperator into Hermitian and anti-Hermitian parts, the former causing pure dissipation while the latter producing coherent effects on the motion. ${ }^{16}$ At this point it is worthwhile to note that in the previous works, ${ }^{1,3,5-13}$ the relaxation superoperator was partitioned into real and imaginary parts. Such a partition is relevant only for the secular terms of the relaxation superoperator. This point is further discussed later. Partitioning into Hermitian and anti-Hermitian is readily made by decomposing the double commutator into parts that are symmetric and antisymmetric in the basis operators. Following Jeener, ${ }^{16}$ we decompose the double commutator as

$$
\begin{aligned}
{\left[\mathcal{S}_{i},\left[\mathcal{S}_{j}, \rho\right]\right]=} & \left\{\left\{\mathcal{S}_{i}, \mathcal{S}_{j}\right\}, \rho\right\}-2\left(\hat{\mathcal{S}}_{i} \times \hat{\mathcal{S}}_{j}+\hat{\mathcal{S}}_{j} \times \hat{\mathcal{S}}_{i}\right) \rho \\
& -\left[\left[\mathcal{S}_{i}, \mathcal{S}_{j}\right], \rho\right],
\end{aligned}
$$

where

$$
\begin{aligned}
& \hat{\mathcal{S}}_{i} \times \hat{\mathcal{S}}_{j} \rho=\mathcal{S}_{i} \rho \mathcal{S}_{j}, \\
& \left\{\mathcal{S}_{i}, \mathcal{S}_{j}\right\}=\mathcal{S}_{i} \mathcal{S}_{j}+\mathcal{S}_{j} \mathcal{S}_{i} .
\end{aligned}
$$

We now define the purely dissipative superoperator $(\hat{\mathcal{R}})$ and the purely coherent superoperator $(\hat{\mathcal{L}})$ as follows:

$$
\begin{aligned}
& \hat{\Gamma}=\hat{\mathcal{R}}+\hat{\mathcal{L}} . \\
& \hat{\mathcal{R}}=\frac{1}{2}\left(\hat{\Gamma}+\hat{\Gamma}^{\dagger}\right), \\
& \hat{\mathcal{L}}=\frac{1}{2}\left(\hat{\Gamma}-\hat{\Gamma}^{\dagger}\right) .
\end{aligned}
$$

The above definition of $\hat{\mathcal{R}}$ and $\hat{\mathcal{L}}$ naturally leads to their being Hermitian and anti-Hermitian respectively. Using the properties

$$
\begin{aligned}
& \left(\hat{\mathcal{S}}_{i} \times \hat{\mathcal{S}}_{j}\right)^{\dagger}=\hat{\mathcal{S}}_{i}^{\dagger} \times \hat{\mathcal{S}}_{j}^{\dagger}, \\
& \left\langle\mathcal{S}_{i}^{\dagger} \mid \mathcal{A}_{l, q}^{\eta \dagger}\right\rangle=\left\langle\mathcal{S}_{i} \mid \mathcal{A}_{l, q}^{\eta}\right\rangle^{*},
\end{aligned}
$$

and

$$
\mathcal{A}_{l, q}^{\eta}=\mathcal{A}_{l,-q}^{\eta \dagger},
$$

we obtain

$$
\begin{aligned}
\hat{\mathcal{R}} \rho= & \frac{1}{2} \sum_{\eta, \eta^{\prime}, l, q, i, j} \mathcal{J}_{l}^{\eta \eta^{\prime}}\left(\omega_{j}\right)\left\langle\mathcal{S}_{i} \mid \mathcal{A}_{l, q}^{\eta}\right\rangle \\
& \times\left\langle\mathcal{S}_{j} \mid \mathcal{A}_{l,-q}^{\eta^{\prime}}\right\rangle\left(\left\{\left\{\mathcal{S}_{i}, \mathcal{S}_{j}\right\}, \rho\right\}\right. \\
& \left.-2\left(\hat{\mathcal{S}}_{i} \times \hat{\mathcal{S}}_{j}+\hat{\mathcal{S}}_{j} \times \hat{\mathcal{S}}_{i}\right) \rho\right), \\
\hat{\mathcal{L}} \rho= & -\frac{1}{2} \sum_{\eta \eta^{\prime}, l, q, i, j} \mathcal{J}_{l}^{\eta \eta^{\prime}}\left(\omega_{j}\right)\left(\mathcal{S}_{i}\left|\mathcal{A}_{l, q}^{\eta}\right\rangle\right. \\
& \times\left\langle\mathcal{S}_{j} \mid \mathcal{A}_{l,-q}^{\eta^{\prime}}\right\rangle\left[\left[\mathcal{S}_{i}, \mathcal{S}_{j}\right], \rho\right] .
\end{aligned}
$$

The commutator structure of the anti-Hermitian part allows us to define a Hamiltonian correction $\mathcal{H}_{\delta}$, by rewriting Eq. (19) as

$$
\hat{\mathcal{L}} \rho=-i\left[\mathcal{H}_{\delta}, \rho\right],
$$

where

$$
\begin{aligned}
\mathcal{H}_{\delta}= & -\frac{i}{2} \sum_{\eta, \eta^{\prime}, l, q, i, j} \mathcal{J}_{l}^{\eta \eta^{\prime}}\left(\omega_{j}\right)\left\langle\mathcal{S}_{i} \mid \mathcal{A}_{l, q}^{\eta}\right\rangle\left\langle\mathcal{S}_{j} \mid \mathcal{A}_{l,-q}^{\eta^{\prime}}\right\rangle \\
& \times\left[\mathcal{S}_{i}, \mathcal{S}_{j}\right] .
\end{aligned}
$$

The anti-Hermitian part of the relaxation superoperator gives rise to a correction to the Hamiltonian. This correction Hamiltonian gives rise to DFS and depends on the complete complex spectral density. It is also nondiagonal in the eigenbasis of the main Hamiltonian, $\mathcal{H}_{s}$. The Hermitian part of the relaxation superoperator is also dependent on the complete complex spectral density and causes pure dissipation and decoherence. The Hermitian and the anti-Hermitian parts have the following symmetry with respect to the trace metric:

$$
\begin{aligned}
& \hat{\mathcal{R}}^{\dagger}=\hat{\mathcal{R}}, \\
& \hat{\mathcal{L}}^{\dagger}=-\hat{\mathcal{L}} .
\end{aligned}
$$

It will be shown in the next section that under the secular approximation wherein the nonresonant terms of the relaxation superoperator are dropped, $\hat{\mathcal{L}}$ and $\hat{\mathcal{R}}$ are dependent on the imaginary and the real parts of the spectral density, respectively. This is also the difference between the present and the earlier treatments of DFS. , $^{1,3,5-13}$

Though we have shown that the master equation describing the relaxation dynamics, has in it, an inherent correction to the coherent dynamics apart from causing dissipation and decoherence and that this correction can be mimiced by a correction to the Hamiltonian, the origin of this similarity has not been presented. Indeed, these corrections have a stigma associated with them, of being dynamic as opposed to the other terms in the Hamiltonian that are more naturally, a property of the spins. In the next section, we present a treatment of the closed system formed by the spins and the lattice. In the lowest order in the interaction between them, they evolve under their free Hamiltonians. The presence of a mutual interaction between them, however, causes a correction to the energy levels of the free spins bath. As a consequence, 
there are corrections to the free Hamiltonian that can be calculated from a standard static perturbation theory. We develop a formalism based on the operator version of static perturbation theory to partially diagonalize the Hamiltonian of the closed system-bath complex. This formalism is shown to give rise to corrections to the Hamiltonian of the spins that we find to be identical with the DFS causing a Hamiltonian correction that we have derived from the master equation. This picture, we believe, is more natural in showing the origin of the Hamiltonian corrections.

\section{BATH-INDUCED CORRECTION TO THE SPIN HAMILTONIAN}

In this section we determine the effective Hamiltonian of a spin system in contact with a heat bath made of the molecular degrees of freedom. We would like to determine the correction to the spin Hamiltonian of the system due to its interaction with the heat bath. In the first order, the average correction to the spin Hamiltonian is zero. In the second order, we find a nonzero residual correction to the spin Hamiltonian. This second-order average Hamiltonian correction is found to be identical with the Hamiltonian correction that mimics the coherent effects of relaxation as modeled by the master equation. We diagonalize the system-bath Hamiltonian to second order in the interaction. We start with the total Hamiltonian of the system-bath complex,

$$
\begin{aligned}
\mathcal{H}_{T} & =\mathcal{H}_{s}+\mathcal{H}_{B}+\lambda \mathcal{H}^{\prime}, \\
& =\mathcal{H}_{0}+\lambda \mathcal{H}^{\prime} .
\end{aligned}
$$

$\mathcal{H}_{s}$ is the spin Hamiltonian, $\mathcal{H}_{B}$ is the Hamiltonian of the bath, and $\lambda$ is a perturbation parameter, which is a measure of the strength of the interaction between them. Assuming that the bath is a macroscopic object at a constant temperature, one can write an effective Hamiltonian for the spin system at various orders in the system-bath interaction. At the lowest order, it is given by

$$
\mathcal{H}_{\delta}^{(1)}=\lambda\left\langle\mathcal{H}^{\prime}\right\rangle \text {. }
$$

The angle brackets imply an averaging over the bath degrees of freedom with respect to the thermal equilibrium bath density operator,

$$
\left\langle\mathcal{H}^{\prime}\right\rangle=\operatorname{Tr}\left(\frac{\exp \left(-\beta \mathcal{H}_{B}\right)}{\mathcal{Z}} \cdot \mathcal{H}^{\prime}\right) .
$$

Substituting for $\mathcal{H}^{\prime}$ from Eq. (2), we have, for the first-order correction to the spin Hamiltonian,

$$
\begin{aligned}
\left\langle\mathcal{H}^{\prime}\right\rangle & =\operatorname{Tr}\left(\frac{\exp \left(-\beta \mathcal{H}_{B}\right)}{\mathcal{Z}} \sum_{\eta, l, q} \chi_{l,-q}^{\eta} \mathcal{A}_{l, q}^{\eta}\right) \\
& =\sum_{\eta, \eta^{\prime}, l, q,} \operatorname{Tr}\left(\frac{\exp \left(-\beta \mathcal{H}_{B}\right)}{\mathcal{Z}} \chi_{l, q}^{\eta}\right) \mathcal{A}_{l, q}^{\eta} .
\end{aligned}
$$

The isotropy of the bath leads to complete averaging of the bath operators as a result of which only rank zero terms could possibly be nonzero. The commonly encountered interactions are tensors of nonzero rank and hence the above correction to the Hamiltonian is zero. The second-order correction to the Hamiltonian can be found by diagonalizing the total Hamiltonian to second order in the system-bath interaction. We construct a unitary operator that diagonalizes the total Hamiltonian to second order, ${ }^{17-19}$

$$
\mathcal{H}_{T}{ }^{D}=\exp (-i S) \mathcal{H}_{T} \exp (i S),
$$

where $S$ is a Hermitian generator of the diagonalization transformation. The diagonality to the second-order condition gives for $S$, the following equation. ${ }^{18,19}$

$$
-i \hat{\Gamma}_{0} S=\mathcal{H}^{\prime} .
$$

This equation can be formally inverted to give for $S$,

$$
S=i \hat{\Gamma}_{0}^{-1} \mathcal{H}^{\prime}
$$

The inverse of the free system-bath Liouvillian is hypothesized to exist based on the observation that there exists an intrinsic decay time of the bath. An integral representation of the inverse can then be given to $S$ as

$$
S=-\int_{0}^{\infty} d \tau \mathcal{H}^{\prime}(\tau),
$$

where

$$
\mathcal{H}^{\prime}(t)=\exp \left(-i \hat{\Gamma}_{0} t\right) \mathcal{H}^{\prime} .
$$

One then has for $\widetilde{\mathcal{H}}_{T}$ the following form up to the second order in the interaction:

$$
\tilde{\mathcal{H}}_{T} \approx \mathcal{H}_{0}+\lambda^{2} \mathcal{H}^{\prime(2)},
$$

where the residual second-order interaction is

$$
\begin{aligned}
\lambda^{2} \mathcal{H}^{\prime(2)} & =-\frac{i}{2}\left[S, \mathcal{H}^{\prime}\right] \\
& =\frac{i}{2} \int_{0}^{\infty} d \tau\left[\mathcal{H}^{\prime}(\tau), \mathcal{H}^{\prime}\right] .
\end{aligned}
$$

We can naturally find the effective Hamiltonian correction to the motion of the spins by averaging the second-order interaction over the equilibrium bath to get

$$
\begin{aligned}
& \mathcal{H}_{\delta}{ }^{(2)}=\left\langle\mathcal{H}^{\prime(2)}\right\rangle \\
& \left\langle\mathcal{H}^{\prime(2)}\right\rangle=-\frac{i}{2} \int_{0}^{\infty} d \tau\left\langle\left[\mathcal{H}^{\prime}, \mathcal{H}^{\prime}(\tau)\right]\right\rangle .
\end{aligned}
$$

Using Eq. (2) and the symmetry of the correlation functions, we find

$$
\begin{aligned}
\left\langle\mathcal{H}^{\prime(2)}\right\rangle= & -\frac{i}{2} \int_{0}^{\infty} d \tau \sum_{\eta \eta^{\prime}, l, l^{\prime}, q, q^{\prime}}\left\langle\left[\chi_{l,-q}^{\eta} \mathcal{A}_{l, q}^{\eta}, \chi_{l^{\prime}, q^{\prime}}^{\eta^{\prime}}\right.\right. \\
& \left.\left.\times(\tau) \mathcal{A}_{l^{\prime},-q^{\prime}}^{\eta^{\prime}}(\tau)\right]\right\rangle \\
= & -\frac{i}{2} \sum_{\eta, \eta^{\prime}, l, q} \int_{0}^{\infty} d \tau\left\{\left\langle\left\{\chi_{l,-q}^{\eta}, \chi_{l, q}^{\eta^{\prime}}(\tau)\right\}\right\rangle\right. \\
& \times\left[\mathcal{A}_{l, q}^{\eta}, \mathcal{A}_{l,-q}^{\eta^{\prime}}(\tau)\right]+\left\langle\left[\chi_{l,-q}^{\eta}, \chi_{l, q}^{\eta^{\prime}}(\tau)\right]\right\rangle \\
& \left.\times\left\{\mathcal{A}_{l, q}^{\eta}, \mathcal{A}_{l,-q}^{\eta^{\prime}}(\tau)\right\}\right\} .
\end{aligned}
$$

We encounter two kinds of correlation functions in the above equation. The antisymmetric correlation (the second term on 
the RHS) function plays a role only when the quantum nature of the bath becomes important. In the present context, however, these effects are negligible and can be dropped. Identifying the symmetric correlation function with the correlation functions that arise in the semiclassical description of the bath in Eq. (3) and using the definition of the earlier defined spectral densities in Eq. (9), we arrive at the expression for the second-order correction to the spin Hamiltonian as

$$
\begin{aligned}
\left\langle\mathcal{H}^{\prime(2)}\right\rangle= & -\frac{i}{2} \sum_{\eta, \eta^{\prime}, l, q, i, j}\left\langle\mathcal{S}_{i} \mid \mathcal{A}_{l, q}^{\eta}\right\rangle\left\langle\mathcal{S}_{j} \mid \mathcal{A}_{l,-q}^{\eta^{\prime}}\right\rangle \\
& \times \mathcal{J}_{l}^{\eta \eta^{\prime}}\left(\omega_{j}\right)\left[\mathcal{S}_{i}, \mathcal{S}_{j}\right] .
\end{aligned}
$$

It is seen that the above correction to the Hamiltonian is identical with the one derived from the master equation. The following comments are in order. It is useful to rewrite the above correction in a manifestly antisymmetric form as follows:

$$
\begin{aligned}
\left\langle\mathcal{H}^{\prime(2)}\right\rangle= & -\frac{i}{4} \sum_{\eta, \eta^{\prime}, l, q, i, j}\left\langle\mathcal{S}_{i} \mid \mathcal{A}_{l, q}^{\eta}\right\rangle\left\langle\mathcal{S}_{j} \mid \mathcal{A}_{l,-q}^{\eta^{\prime}}\right\rangle \\
& \times\left\{\mathcal{J}_{l}^{\eta \eta^{\prime}}\left(\omega_{j}\right)-\mathcal{J}_{l}^{\eta \eta^{\prime}}\left(\omega_{i}\right)\right\}\left[\mathcal{S}_{i}, \mathcal{S}_{j}\right] .
\end{aligned}
$$

The above Hamiltonian is seen to depend upon the complete complex spectral densities. From the symmetry relations of the complex spectral densities equation (10), it is evident that the secular part of the above correction $\left(\omega_{i}=-\omega_{j}\right)$ is dependent on the imaginary part of the spectral density alone. A similar argument for the Hermitian part of the relaxation superoperator in Eq. (18) shows that under the same approximation, it is a function of the real part of the spectral density alone. In the Appendix, we give the matrix representation of the Hermitian and the anti-Hermitian parts of the relaxation superoperator in the case of a system of two spins- $\frac{1}{2}$. There, it is clearly seen that contributions from the complete complex spectral densities are only to the off-diagonal elements.

The secular part of the above correction $\left(\left\langle\mathcal{H}^{\prime(2)}\right\rangle_{0}\right.$, is given as

$$
\begin{aligned}
\left\langle\mathcal{H}_{0}^{\prime(2)}\right\rangle= & -\frac{i}{2} \sum_{\eta, \eta^{\prime}, l, q, i, j}\left\langle\mathcal{S}_{i} \mid \mathcal{A}_{l, q}^{\eta}\right\rangle\left\langle\mathcal{S}_{j} \mid \mathcal{A}_{l,-q}^{\eta^{\prime}}\right\rangle \\
& \times \mathcal{J}_{l}^{\eta \eta^{\prime}}\left(\omega_{j i}\right)\left[\mathcal{S}_{i}, \mathcal{S}_{j}\right]
\end{aligned}
$$

where $\omega_{j i}=\frac{1}{2}\left(\omega_{j}-\omega_{i}\right)$. In the above sum, the $i$ and $j$ are restricted such that $\omega_{i}+\omega_{j}=0$. After some algebra we find, for the shifts,

$$
\begin{aligned}
\left\langle\mathcal{H}_{0}^{\prime(2)}\right\rangle= & -\frac{i}{4} \sum_{\eta, \eta^{\prime}, l, q, i, j}\left\langle\mathcal{S}_{i} \mid \mathcal{A}_{l, q}^{\eta}\right\rangle\left\langle\mathcal{S}_{j} \mid \mathcal{A}_{l,-q}^{\eta^{\prime}}\right\rangle\left(\mathcal{J}_{l}^{\eta \eta^{\prime}}\left(\omega_{j i}\right)\right. \\
& \left.-\mathcal{J}_{l}^{\eta \eta^{\prime}}\left(\omega_{i j}\right)\right)\left[\mathcal{S}_{i}, \mathcal{S}_{j}\right] \\
= & \frac{1}{2} \sum_{\eta, \eta^{\prime}, l, q, i, j}\left\langle\mathcal{S}_{i} \mid \mathcal{A}_{l, q}^{\eta}\right\rangle\left\langle\mathcal{S}_{j} \mid \mathcal{A}_{l,-q}^{\eta^{\prime},}\right\rangle K_{l}^{\eta \eta^{\prime}}\left(\omega_{j i}\right) \\
& \times\left[\mathcal{S}_{i}, \mathcal{S}_{j}\right] .
\end{aligned}
$$

In the above equation, $K_{l}^{\eta \eta^{\prime}}(\omega)$ are the imaginary part of the spectral densities that are antisymmetric functions of the frequency and are given by

$$
K_{l}^{\eta \eta^{\prime}}(\omega)=\int_{0}^{\infty} \sin (\omega \tau) \mathcal{C}_{l}^{\eta \eta^{\prime}}(\tau) d \tau
$$

Equation (41) is identical with the Hamiltonian derived in Ref. 11. However, the same form for the correction Hamiltonian was assumed to hold beyond the secular approximation. In this, the present treatment differs. We have shown that the DFS arises from the secular part of a more general correction to the spin Hamiltonian induced by the interaction with the bath.

\section{THE HAMILTONIAN CORRECTIONS IN PARTICULAR SPIN-SYSTEMS}

We now examine the shift producing Hamiltonians in a few interesting cases.

(1) A system of two spins- $\frac{1}{2}$ : The possible relaxation Hamiltonian of a system of two spins $-\frac{1}{2}$ is given by

$$
\mathcal{H}^{\prime}=\mathcal{H}_{A}{ }^{\mathrm{CSA}}+\mathcal{H}_{M}{ }^{\mathrm{CSA}}+\mathcal{H}_{A M}{ }^{\mathrm{DD}}
$$

where the first two terms on the RHS are the chemical shift anisotropies associated with the two spins and the third term is the mutual dipolar interaction between them. These are defined further as in Eq. (2) with

$$
\begin{aligned}
& A_{0}=\sqrt{\frac{2}{3}} A_{Z}, \quad A_{ \pm 1}=\frac{1}{2} A_{ \pm}, \\
& (A \otimes M)_{0}=\frac{1}{\sqrt{6}}\left(2 A_{Z} M_{Z}-\frac{1}{2}\left(A_{+} M_{-}+A_{-} M_{+}\right)\right),
\end{aligned}
$$

$$
\begin{aligned}
& (A \otimes M)_{ \pm 1}=\frac{1}{2}\left(A_{ \pm} M_{Z}+A_{Z} M_{ \pm}\right), \\
& (A \otimes M)_{ \pm 2}=\frac{1}{2} A_{ \pm} M_{ \pm} .
\end{aligned}
$$

Using the above expressions in Eq. (39), we find for the total correction to the Hamiltonian as 


$$
\begin{aligned}
\left\langle\mathcal{H}^{\prime(2)}\right\rangle= & -\left[\frac{1}{2} K_{2}^{A, A}\left(\omega_{A}\right)+\frac{1}{8} K_{2}^{A M, A M}\left(\omega_{A}\right)+\frac{1}{4} K_{2}^{A M, A M}\left(\omega_{A}+\omega_{M}\right)+\frac{1}{24} K_{2}^{A M, A M}\left(\omega_{A}-\omega_{M}\right)\right] A_{Z}-\left[\frac{1}{2} K_{2}^{M, M}\left(\omega_{M}\right)+\frac{1}{8} K_{2}^{A M, A M}\left(\omega_{M}\right)\right. \\
& \left.+\frac{1}{4} K_{2}^{A M, A M}\left(\omega_{A}+\omega_{M}\right)-\frac{1}{24} K_{2}^{A M, A M}\left(\omega_{A}-\omega_{M}\right)\right] M_{Z}-\left[K_{2}^{A, A M}\left(\omega_{A}\right)+K_{2}^{M, A M}\left(\omega_{M}\right)\right] A_{Z} M_{Z} \\
& -\left[\frac{1}{12}\left\{K_{2}^{A, A M}\left(\omega_{A}-\omega_{M}\right)-K_{2}^{M, A M}\left(\omega_{A}-\omega_{M}\right)\right\}+\frac{1}{4}\left\{K_{2}^{A, A M}\left(\omega_{M}\right)+K_{2}^{A, A M}\left(\omega_{A}\right)+K_{2}^{M, A M}\left(\omega_{M}\right)+K_{2}^{M, A M}\left(\omega_{A}\right)\right\}\right] \\
& \times\left(A_{+} M_{-}+A_{-} M_{+}\right)+i\left[\frac{1}{12}\left\{J_{2}^{A, A M}\left(\omega_{A}-\omega_{M}\right)-J_{2}^{A, A M}(0)-J_{2}^{M, A M}\left(\omega_{A}-\omega_{M}\right)+J_{2}^{M, A M}(0)\right\}\right. \\
& \left.+\frac{1}{4}\left\{J_{2}^{A, A M}\left(\omega_{M}\right)-J_{2}^{A, A M}\left(\omega_{A}\right)+J_{2}^{M, A M}\left(\omega_{M}\right)-J_{2}^{M, A M}\left(\omega_{A}\right)\right\}\right]\left(A_{+} M_{-}-A_{-} M_{+}\right) .
\end{aligned}
$$

In the above equation, $K_{2}^{A, A}$ and $K_{2}^{M, M}$ are the autocorrelation spectral densities, respectively, of the CSA of the spins $A$ and $M, K_{2}^{A M, A M}$ are the autocorrelation spectral densities of the dipolar interaction and $K_{2}^{A, A M}$ and $K_{2}^{M, A M}$ are the crosscorrelation spectral densities, respectively, between the CSA of spins $A$ and $M$, and dipolar interaction. $J_{2}^{\eta, \eta^{\prime}}$ are the real part of the spectral densities that are symmetric functions of the frequency and are given as

$$
J_{l}^{\eta, \eta^{\prime}}=\int_{0}^{\infty} \cos (\omega \tau) \mathcal{C}_{l}^{\eta \eta^{\prime}}(\tau) d \tau .
$$

The diagonal part of the above corrective Hamiltonian [the first three terms on the RHS of Eq. (45)] are seen to depend only on the imaginary part of the spectral densities while the off-diagonal part is found to be complex, in general (also see the Appendix). For homonuclear spins $\left(\left|\omega_{A}-\omega_{M}\right| \tau_{c} \ll 1\right)$, the spectral densities at the two Larmor frequencies are equal. The contributions from the real part of the spectral densities are seen to tend to zero in this limit, thus making the DFS arise solely from the imaginary part of the spectral densities. The above Hamiltonian is then seen to take the following form:

$$
\left\langle\mathcal{H}^{\prime(2)}\right\rangle=-\Omega_{A} A_{Z}-\Omega_{M} M_{Z}-\Delta \mathbf{A} \cdot \mathbf{M},
$$

where $\Delta$ is the relaxation induced coupling between the spins and is given by

$$
\Delta=K_{2}^{A, A M}\left(\omega_{A}\right)+K_{2}^{M, A M}\left(\omega_{M}\right),
$$

and the Larmor frequency shifts are given by the following expressions:

$$
\begin{aligned}
\Omega_{A}= & \left\{\frac{1}{2} K_{2}^{A, A}\left(\omega_{A}\right)+\frac{1}{8} K_{2}^{A M, A M}\left(\omega_{A}\right)\right. \\
& \left.+\frac{1}{4} K_{2}^{A M, A M}\left(\omega_{A}+\omega_{M}\right)\right\}
\end{aligned}
$$

and

$$
\begin{aligned}
\Omega_{M}= & \left\{\frac{1}{2} K_{2}^{M, M}\left(\omega_{M}\right)+\frac{1}{8} K_{2}^{A M, A M}\left(\omega_{M}\right)\right. \\
& \left.+\frac{1}{4} K_{2}^{A M, A M}\left(\omega_{A}+\omega_{M}\right)\right\} .
\end{aligned}
$$

For homonuclear systems, therefore, the total spin Hamiltonian $\mathcal{H}_{T}^{S}$ can be written (including the relaxation-induced couplings and Larmor frequency shifts) as

$$
\mathcal{H}_{T}^{S}=-\left(\omega_{A}+\Omega_{A}\right) A_{Z}-\left(\omega_{M}+\Omega_{M}\right) M_{Z}+(J-\Delta) \mathbf{A} \cdot \mathbf{M} .
$$

In the limit of weak coupling between the spins $\left|\omega_{A}-\omega_{M}\right|$ $\gg \Delta$, the Hamiltonian correction takes the following form: ${ }^{10}$

$$
\left\langle\mathcal{H}^{\prime(2)}\right\rangle=-\Omega_{A} A_{Z}-\Omega_{M} M_{Z}-\Delta A_{Z} M_{Z} .
$$

It is to be noted that even when the Larmor frequencies of the spins are equal, the relaxation-induced corrections to the Larmor frequencies could be different when the anisotropy of the chemical shifts to the spins are different.

The above example clearly demonstrates the fact that the real part of the spectral densities play only a negligible role in causing frequency shifts. This point is further illustrated in the Appendix using the matrix representation of the Hermitian and the anti-Hermitian parts of the relaxation superoperator. In what follows, we neglect the real part.

(2) Cross-correlation-induced three-spin coupling: We consider a system of three spins- $\frac{1}{2}$ interacting via the magnetic dipolar interaction. As in Ref. 11, we arrive at the following expression for the relaxation-induced coupling Hamiltonian:

$$
\left\langle\mathcal{H}^{\prime(2)}\right\rangle=\mathcal{H}_{A}+\mathcal{H}_{M}+\mathcal{H}_{X},
$$

where

$$
\begin{aligned}
\mathcal{H}_{A}= & -\frac{1}{2} K_{2}^{A M, A X}\left(\omega_{A}\right) A_{Z} M_{Z} X_{Z}+\frac{1}{8}\left(K_{2}^{A M, A X}\left(\omega_{M}\right)+K_{2}^{A M, A X}\left(\omega_{A}\right)\left(A_{+} M_{-}+A_{-} M_{+}\right) X_{Z}+\frac{1}{8}\left(K_{2}^{A M, A X}\left(\omega_{X}\right)\right.\right. \\
& \left.+K_{2}^{A M, A X}\left(\omega_{A}\right)\right)\left(A_{+} X_{-}+A_{-} X_{+}\right) M_{Z}-\frac{1}{4}\left(K_{2}^{A M, A X}\left(\omega_{X}+\omega_{A}\right)+K_{2}^{A M, A X}\left(\omega_{M}+\omega_{A}\right)\right)\left(X_{+} M_{-}+X_{-} M_{+}\right) A_{Z},
\end{aligned}
$$

with similar expressions for $\mathcal{H}_{M}$ and $\mathcal{H}_{X}$ with appropriate changes in the indices. Again, for homonuclear spins $\left(\mid \omega_{A}\right.$ $-\omega_{M}\left|\tau_{c} \approx\right| \omega_{M}-\omega_{X} \mid \tau_{c} \ll 1$, we can replace all the arguments of the spectral densities $\omega_{A} \approx \omega_{M} \approx \omega_{X}=\omega$. We then have ${ }^{11}$

$$
\begin{aligned}
\left\langle\mathcal{H}^{\prime(2)}\right\rangle= & -\frac{1}{2}\left(K_{2}^{A M, A X}(\omega)+K_{2}^{A M, M X}(\omega)+K_{2}^{A X, M X}(\omega)\right) A_{Z} M_{Z} X_{Z}+\frac{1}{2}\left(K_{2}^{A M, A X}(\omega)+K_{2}^{A M, M X}(\omega)\right. \\
& \left.+K_{2}^{A X, M X}(2 \omega)\right)\left(A_{+} M_{-}+A_{-} M_{+}\right) X_{Z}+\frac{1}{2}\left(K_{2}^{A M, A X}(\omega)+K_{2}^{A X, M X}(\omega)+K_{2}^{A M, M X}(2 \omega)\right)\left(A_{-} X_{+}+A_{+} X_{-}\right) M_{Z} \\
& +\frac{1}{2}\left(K_{2}^{A M, M X}(\omega)+K_{2}^{A X, M X}(\omega)+K_{2}^{A M, A X}(2 \omega)\right)\left(X_{+} M_{-}+X_{-} M_{+}\right) A_{Z} .
\end{aligned}
$$


In the case of weak coupling, the terms that cause mixing between the various spin states are rendered ineffective, and we arrive at a weak coupling Hamiltonian given by ${ }^{11}$

$$
\left\langle\mathcal{H}^{\prime(2)}\right\rangle=-\frac{1}{2}\left(K_{2}^{A M, A X}\left(\omega_{A}\right)+K_{2}^{A M, M X}\left(\omega_{M}\right)+K_{2}^{A M, M X}\left(\omega_{X}\right)\right) A_{Z} M_{Z} X_{Z}
$$

The implications of such a coupling term have been dealt with earlier ${ }^{11}$ and will not be treated here. It may be pointed out that the autocorrelations [not included in Eq. (55)] also give rise to DFS, which cause unequal Larmor frequency shifts on the various spins.

(3) Quadrupolar-DD cross-correlation-induced Hamiltonian shifts: We consider a spin- $\frac{1}{2}(I)-$ spin $>\frac{1}{2}(S)$ system relaxing via their mutual dipolar interaction $(I S)$ and the quadrupolar interaction $\left(Q_{S}\right)$ of the spin $>\frac{1}{2}$. The relaxation-induced coupling between them arises from both the autocorrelation of the dipolar interaction $\left(K_{2}^{I S, I S}\right)$ and the cross-correlation between the dipolar interaction and the quadrupolar interaction ${ }^{5-8,12}\left(K_{2}^{Q_{S}, I S}\right)$ giving the following coupling Hamiltonian.

$$
\begin{aligned}
\left\langle\mathcal{H}^{\prime(2)}\right\rangle= & -\left[\frac{1}{2} K_{2}^{I S, I S}\left(\omega_{I}\right)+2 K_{2}^{Q_{S}, I S}\left(\omega_{S}\right)\right] S_{Z}^{2} I_{Z}+\frac{1}{16}\left[K_{2}^{I S, I S}\left(\omega_{S}\right)+K_{2}^{I S, I S}\left(\omega_{I}\right)+K_{2}^{Q_{S}, I S}\left(\omega_{S}\right)+K_{2}^{Q_{S}, I S}\left(\omega_{I}\right)\right. \\
& \left.-4 K_{2}^{Q_{S}, I S}\left(\omega_{S}+\omega_{I}\right)-4 K_{2}^{Q_{S}, I S}\left(2 \omega_{S}\right)\right]\left[I_{-}\left\{S_{+}, S_{Z}\right\}+I_{+}\left\{S_{-}, S_{Z}\right\}\right]+\left[K_{2}^{Q_{S}, I S}\left(\omega_{S}\right)-\frac{1}{12} K_{2}^{I S, I S}\left(\omega_{I}-\omega_{S}\right)\right. \\
& \left.-\frac{1}{2} K_{2}^{I S, I S}\left(\omega_{I}+\omega_{S}\right)\right]\left[I_{Z}\left(S^{2}-S_{Z}^{2}\right)\right] .
\end{aligned}
$$

A feature in the above Hamiltonian is the coupling of the spin- $\frac{1}{2}$ to the $\operatorname{spin}>\frac{1}{2}$, which breaks the symmetry of the multiplet structure of the spin- $\frac{1}{2}$. In the limit $\left|\omega_{I}-\omega_{S}\right|$ $\gg K_{2}^{Q_{S}, I S}, K_{2}^{I S, I S}$, the second term is rendered ineffective. The above coupling then takes the following form:

$$
\begin{aligned}
\left\langle\mathcal{H}^{\prime(2)}\right\rangle= & -\left[\frac{1}{2} K_{2}^{I S, I S}\left(\omega_{I}\right)+K_{2}^{I S, I S}\left(\omega_{I}-\omega_{S}\right)\right. \\
& \left.+K_{2}^{I S, I S}\left(\omega_{I}+\omega_{S}\right)\right] I_{Z} S_{Z}^{2}+\left[K_{2}^{Q_{S}, I S}\left(\omega_{S}\right)\right. \\
& \left.-\frac{1}{12} K_{2}^{I S, I S}\left(\omega_{I}-\omega_{S}\right)-\frac{1}{2} K_{2}^{I S, I S}\left(\omega_{I}+\omega_{S}\right)\right] \\
& \times\left[I_{Z}\left(S^{2}-3 S_{Z}^{2}\right)\right]
\end{aligned}
$$

The above Hamiltonian also presents the possibility of the mutual cancellation of the effects produced by the dipolar autocorrelation and the quadrupolar-dipolar crosscorrelation terms in breaking the symmetry of the multiplet structure of the spin- $\frac{1}{2}$. The autocorrelation of the quadrupolar interaction also produces DFS that can be observed and are presented in the next section.

(4) Hamiltonian shifts induced by fluctuating quadrupolar interaction: Here we consider a lone spin $>\frac{1}{2}$ relaxed by the electric quadrupolar interaction. ${ }^{8}$ The Hamiltonian shifts produced by the autocorrelation of the quadrupolar interaction is given by

$$
\begin{aligned}
\left\langle\mathcal{H}^{\prime(2)}\right\rangle= & \frac{1}{2}\left\{2 K_{2}^{Q_{S}}\left(2 \omega_{S}\right)-K_{2}^{Q_{S}}\left(\omega_{S}\right)\right\} S_{Z}^{3} \\
& -\left(S^{2}-1\right) K_{2}^{Q_{S}}\left(2 \omega_{S}\right) S_{Z} .
\end{aligned}
$$

The second term in the above correction causes equal shifts to all the transitions while the first term (for $S>1$ ), lifts the degeneracy of all the Zeeman transitions and hence produces satellites. The effect of these shifts is expected to be greater than all the other known shifts because of their dependence on the autocorrelation functions rather than the crosscorrelation functions. It is to be noted that these shifts become observable only for spin $>1$. For example, in the case of a spin $\left(S=\frac{3}{2}\right)$, the $\frac{1}{2},-\frac{1}{2}$ transition is shifted less than the $\frac{3}{2}, \frac{1}{2}$ and the $-\frac{1}{2},-\frac{3}{2}$ transitions yielding a satellite formation, the splitting being $\frac{1}{8}\left(2 K_{2}^{Q_{S}}\left(2 \omega_{S}\right)-K_{2}^{Q_{S}}\left(\omega_{S}\right)\right)$.

\section{DISCUSSION AND CONCLUSION}

We have shown that the relaxation superoperator can be partitioned into Hermitian and anti-Hermitian parts that cause purely dissipative and purely coherent effects on the motion of the system. The latter was shown to possess a commutator structure that lead us to define a general Hamiltonian correction to the system. Earlier works decomposed the relaxation superoperator into real and imaginary parts. We have shown that it is more instructive to decompose it into a Hermitian and an anti-Hermitian part. Within the secular approximation, the latter decomposition is found to be identical with the former. As a result of the present decomposition, both coherent and dissipative effects of the relaxation are seen to depend on the complete complex spectral densities. Again, in the secular limit, the coherent effects are seen to depend exclusively on the imaginary part while the dissipative effects are seen to depend on the real part of the spectral densities. To provide a physical basis to the above arguments, the correction to the system Hamiltonian was determined to the lowest nonzeroth order in the system-bath interaction. It was shown that the second-order correction to the system Hamiltonian naturally arises out of a perturbative diagonalization procedure, and this correction was found to be identical with the Hamiltonian correction extracted from the master equation. The correction was found to have both diagonal and off-diagonal elements. Under the secular approximation, the influence of the off-diagonal terms are negligible and the resulting Hamiltonian correction only causes first-order DFS, and was shown to depend, in agreement with the literature, only on the imaginary part of the spectral densities. The nonsecular parts of the DFS Hamiltonian can give rise to observable features the case of rf relaxation in the presence of irradiation (to be published). It is interesting to note that the role of the real part of the spectral densities in causing coherent evolution is small and often negligible. The reason for this can be traced back to the scalar nature of the relaxation causing a Hamiltonian. When this scalar nature is broken, the effects of the real part of the spectral densities is 
expected to be important. The well-known two- and threespin $\mathrm{J}$ couplings that arise due to relaxation, were shown to arise out of this formalism. An instance where the autocorrelation functions give rise to observable shifts was demonstrated for a lone spin $>1$. To conclude, we have shown that the DFS can always be thought of as arising out of a correction to the Hamiltonian and a formalism has been provided that naturally leads to the determination of these Hamiltonians. It has been shown that the general DFS Hamiltonian depends on the complete complex spectral densities and that the first-order DFS depends only on the imaginary part of the spectral densities. The same feature is noted in the case of the dissipative part of the relaxation too. We find that the imaginary part of the spectral densities do contribute to the dissipation. However, they always contribute only to the nonsecular part.

\section{APPENDIX: MATRIX REPRESENTATION OF THE HERMITIAN AND THE ANTI-HERMITIAN PARTS OF THE RELAXATION SUPEROPERATOR OF A SYSTEM OF TWO SPINS- $\frac{1}{2}$}

Our purpose in this appendix is to show explicitly the matrix structure of the Hermitian and the anti-Hermitian parts of the relaxation superoperator. We consider a system of two spins $-\frac{1}{2}$. The eigenstates of the spin Hamiltonian are labeled as $1 \longrightarrow|\uparrow \uparrow\rangle, 2 \rightarrow|\downarrow \uparrow\rangle, 3 \rightarrow|\uparrow \downarrow\rangle, 4 \longrightarrow|\downarrow \downarrow\rangle$. The corresponding basis superkets spanning the space of single quantum transitions can be labeled as $|1\rangle\langle 2|| 3\rangle,\langle 4|| 1\rangle,\langle 3|$, and $|2\rangle\langle 4|$. In this basis, the total coherent superoperator can be written as

$$
\hat{\mathcal{H}}=-i\left(\begin{array}{cccc}
-\nu_{A}-\frac{1}{2} \eta & 0 & \delta-i \gamma & 0 \\
0 & -\nu_{A}+\frac{1}{2} \eta & 0 & \delta-i \gamma \\
\delta+i \gamma & 0 & -\nu_{M}-\frac{1}{2} \eta & 0 \\
0 & \delta+i \gamma & 0 & -\nu_{M}+\frac{1}{2} \eta
\end{array}\right) \text {, }
$$

where

$$
\begin{aligned}
\nu_{A}= & \omega_{A}+\left[\frac{1}{2} K_{2}^{A, A}\left(\omega_{A}\right)+\frac{1}{8} K_{2}^{A M, A M}\left(\omega_{A}\right)\right. \\
& \left.+\frac{1}{4} K_{2}^{A M, A M}\left(\omega_{A}+\omega_{M}\right)+\frac{1}{24} K_{2}^{A M, A M}\left(\omega_{A}-\omega_{M}\right)\right], \\
\nu_{M}= & \omega_{M}+\left[\frac{1}{2} K_{2}^{M, M}\left(\omega_{M}\right)+\frac{1}{8} K_{2}^{A M, A M}\left(\omega_{M}\right)\right. \\
& \left.+\frac{1}{4} K_{2}^{A M, A M}\left(\omega_{A}+\omega_{M}\right)-\frac{1}{24} K_{2}^{A M, A M}\left(\omega_{A}-\omega_{M}\right)\right], \\
\eta= & +\left[K_{2}^{A, A M}\left(\omega_{A}\right)+K_{2}^{M, A M}\left(\omega_{M}\right)\right], \\
\delta= & {\left[\frac{1}{12}\left\{K_{2}^{A, A M}\left(\omega_{A}-\omega_{M}\right)-K_{2}^{M, A M}\left(\omega_{A}-\omega_{M}\right)\right\}\right.} \\
& +\frac{1}{4}\left\{K_{2}^{A, A M}\left(\omega_{M}\right)+K_{2}^{A, A M}\left(\omega_{A}\right)+K_{2}^{M, A M}\left(\omega_{M}\right)\right. \\
& \left.\left.+K_{2}^{M, A M}\left(\omega_{A}\right)\right\}\right], \\
\gamma= & {\left[\frac { 1 } { 1 2 } \left\{J_{2}^{A, A M}\left(\omega_{A}-\omega_{M}\right)-J_{2}^{A, A M}(0)-J_{2}^{M, A M}\left(\omega_{A}-\omega_{M}\right)\right.\right.} \\
& \left.+J_{2}^{M, A M}(0)\right\}+\frac{1}{4}\left\{J_{2}^{A, A M}\left(\omega_{M}\right)-J_{2}^{A, A M}\left(\omega_{A}\right)\right. \\
& \left.\left.+J_{2}^{M, A M}\left(\omega_{M}\right)-J_{2}^{M, A M}\left(\omega_{A}\right)\right\}\right] .
\end{aligned}
$$

The first-order DFS are contained in $\eta$, which modifies the $\mathrm{J}$ coupling between spins. This term is in agreement with Tjandra et al. ${ }^{9}$ and Brüschweiler. ${ }^{10}$ The off-diagonal terms of the matrix $(\delta \pm i \gamma)$ connect the transitions of different spins and yield second-order shifts $\left(\delta^{2}+\gamma^{2}\right) /\left(\nu_{A}-\nu_{M}\right)$ to the frequencies. These shifts are negligible when the Larmor frequencies of the two spins are sufficiently different $\left[\left(\delta^{2}+\gamma^{2}\right) / \mid \nu_{A}\right.$
$\left.-\left.\nu_{M}\right|^{2} \ll 1\right]$. When the transitions of the spins become degenerate, $\gamma$ goes to zero but $\delta$ does not. Hence, in both the extreme limits, the real part of the spectral densities are rendered ineffective. In the intermediate region, both the real and the imaginary parts of the spectral densities contribute to the DFS. This feature has not been reported earlier to the best of our knowledge.

The Hermitian part of the relaxation superoperator can also be given a similar matrix representation in a suitable basis of single quantum operators defined as follows: 1 $\rightarrow(1 / \sqrt{2}) A_{+}, \quad 2 \rightarrow \sqrt{2} A_{+} M_{Z}, \quad 3 \rightarrow(1 / \sqrt{2}) M_{+}, \quad$ and $\quad 4$ $\rightarrow \sqrt{2} M_{+} A_{Z}$. In this basis, the Hermitian part takes the form

$$
\hat{\mathcal{R}}=\left(\begin{array}{cccc}
\rho_{A_{+}} & \delta_{A} & \Delta_{A M} & \eta_{A} \\
\delta_{A} & \rho_{A_{+} M_{Z}} & \eta_{M} & \Delta_{A M} \\
\Delta_{A M}^{*} & \eta_{M}^{*} & \rho_{M_{+}} & \delta_{M} \\
\eta_{A}^{*} & \Lambda_{A M}^{*} & \delta_{M}^{*} & \rho_{M_{+} A_{Z}}
\end{array}\right),
$$

where the elements are defined as

$$
\begin{aligned}
\rho_{A_{+}}= & \frac{1}{2} J_{2}^{A, A}\left(\omega_{A}\right)+\frac{2}{3} J_{2}^{A, A}(0)+\frac{1}{8} J_{2}^{A M, A M}\left(\omega_{A}\right) \\
& +\frac{1}{6} J_{2}^{A M, A M}(0)+\frac{1}{24} J_{2}^{A M, A M}\left(\omega_{A}-\omega_{M}\right) \\
& +\frac{1}{2} J_{2}^{A M, A M}\left(\omega_{A}+\omega_{M}\right)+\frac{1}{4} J_{2}^{A M, A M}\left(\omega_{M}\right),
\end{aligned}
$$




$$
\begin{aligned}
& \rho_{M_{+}}=\frac{1}{2} J_{2}^{M, M}\left(\omega_{M}\right)+\frac{2}{3} J_{2}^{M, M}(0)+\frac{1}{8} J_{2}^{A M, A M}\left(\omega_{M}\right) \\
& +\frac{1}{6} J_{2}^{A M, A M}(0)+\frac{1}{24} J_{2}^{A M, A M}\left(\omega_{A}-\omega_{M}\right) \\
& +\frac{1}{2} J_{2}^{A M, A M}\left(\omega_{A}+\omega_{M}\right)+\frac{1}{4} J_{2}^{A M, A M}\left(\omega_{A}\right), \\
& \rho_{A_{+} M_{Z}}=\frac{1}{2} J_{2}^{A, A}\left(\omega_{A}\right)+\frac{2}{3} J_{2}^{A, A}(0)+\frac{1}{8} J_{2}^{A M, A M}\left(\omega_{A}\right) \\
& +\frac{1}{6} J_{2}^{A M, A M}(0)+\frac{1}{4} J_{2}^{A M, A M}\left(\omega_{A}+\omega_{M}\right) \\
& +\frac{1}{24} J_{2}^{A M, A M}\left(\omega_{A}-\omega_{M}\right), \\
& \rho_{M_{+} A_{Z}}=\frac{1}{2} J_{2}^{M, M}\left(\omega_{M}\right)+\frac{2}{3} J_{2}^{M, M}(0)+\frac{1}{8} J_{2}^{A M, A M}\left(\omega_{M}\right) \\
& +\frac{1}{6} J_{2}^{A M, A M}(0)+\frac{1}{2} J_{2}^{A M, A M}\left(\omega_{A}+\omega_{M}\right) \\
& +\frac{1}{24} J_{2}^{A M, A M}\left(\omega_{M}-\omega_{A}\right), \\
& \delta_{A}=\frac{1}{2} J_{2}^{A, A M}\left(\omega_{A}\right)+\frac{2}{3} J_{2}^{A, A M}(0), \\
& \delta_{M}=\frac{1}{2} J_{2}^{M, A M}\left(\omega_{M}\right)+\frac{2}{3} J_{2}^{M, A M}(0), \\
& \Delta_{A M}=-\frac{1}{12}\left(\mathcal{J}_{2}^{A M, A M}(0)+\mathcal{J}_{2}^{A M, A M}\left(\omega_{A}-\omega_{M}\right)\right) \\
& +\frac{1}{8}\left(\mathcal{J}_{2}^{A M, A M}\left(\omega_{A}\right)+\mathcal{J}_{2}^{A M, A M}\left(-\omega_{M}\right)\right), \\
& \eta_{A}=-\frac{1}{6}\left(\mathcal{J}_{2}^{A, A M}(0)+\mathcal{J}_{2}^{A, A M}\left(\omega_{A}-\omega_{M}\right)\right) \\
& +\frac{1}{4}\left(\mathcal{J}_{2}^{A, A M}\left(\omega_{A}\right)+\mathcal{J}_{2}^{A, A M}\left(-\omega_{M}\right)\right), \\
& \eta_{M}=-\frac{1}{6}\left(\mathcal{J}_{2}^{M, A M}(0)+\mathcal{J}_{2}^{M, A M}\left(\omega_{A}-\omega_{M}\right)\right) \\
& +\frac{1}{4}\left(\mathcal{J}_{2}^{M, A M}\left(-\omega_{M}\right)+\mathcal{J}_{2}^{A, A M}\left(\omega_{A}\right)\right), \\
& \Lambda_{A M}=\frac{1}{12}\left(\mathcal{J}_{2}^{A M, A M}(0)+\mathcal{J}_{2}^{A M, A M}\left(\omega_{A}-\omega_{M}\right)\right) .
\end{aligned}
$$

In the above matrix, we find that the terms that connect the transverse orders of the different spins $(\Delta, \eta$, and $\Lambda)$ are complex while the terms that connect the various transverse orders of a spin $(\delta)$ are real. The real part of the above matrix agrees with the calculations presented earlier in the literature. ${ }^{13,20}$ Again, in the limit when the Larmor frequencies of the two spins are equal, wherein we would expect these complex terms to be effective, the contribution to these terms from the imaginary part of the spectral densities tend to zero, thus making the imaginary part of the spectral densities, practically ineffective in contributing to the incoherent evolution.

Hence, we note that though, in principle, both the real and the imaginary parts of the spectral densities contribute to the DFS and the decoherence, the role played by the real and the imaginary parts of the spectral densities in causing DFS and decoherence, respectively, is rendered ineffective in the extreme cases of complete resolution and complete degeneracy of the lines. In the intermediate region, the complete complex matrices corresponding to the DFS and decoherence, should be used. The above treatment of the relaxation of a system of two spins- $\frac{1}{2}$ is more general within the Redfield relaxation theory and to the best of our knowledge never been presented in the literature.

${ }^{1}$ A. G. Redfield, Adv. Magn. Reson. 1, 1 (1965).

${ }^{2}$ R. Kubo and K. Tomita, J. Phys. Soc. Jpn. 9, 889 (1954).

${ }^{3}$ A. Abragam, The Principles of Nuclear Magnetism (Clarendon, Oxford, 1961).

${ }^{4}$ J. McConnell, The Theory of Nuclear Spin Relaxation in Liquids (Cambridge University Press, Cambridge, 1987).

${ }^{5}$ R. E. London, D. M. LeMaster, and L. G. Werbelow, J. Am. Chem. Soc. 116, 8400 (1994).

${ }^{6}$ L. G. Werbelow, in The Encyclopedia of NMR, edited by D. M. Grant and R. K. Harris (Wiley, New York, 1996), p. 4092.

${ }^{7}$ L. G. Werbelow and R. E. London, J. Chem. Phys. 102, 5181 (1995).

${ }^{8}$ L. G. Werbelow and R. E. London, Concepts Magn. Reson. 8, 325 (1996).

${ }^{9}$ N. Tjandra, S. Grzesiek, and A. Bax, J. Am. Chem. Soc. 118, 6264 (1996).

${ }^{10} \mathrm{R}$. Brüschweiler, Chem. Phys. Lett. 257, 119 (1996).

${ }^{11}$ R. Brüschweiler, J. Chem. Phys. 105, 6164 (1996).

${ }^{12}$ L. G. Werbelow and J. Kowalewski, J. Chem. Phys. 107, 2775 (1997).

${ }^{13}$ A. Kumar, R. C. Rani Grace, and P. K. Madhu, "Cross correlations in NMR,' Prog. NMR Spectrosc. 37, 191 (2000).

${ }^{14}$ V. Romero-Rochin and I. Oppenheim, Physica A 155, 52 (1989).

${ }^{15}$ J. Jeener, Adv. Magn. Reson. 10, 1 (1982).

${ }^{16}$ M. Mehring, Principles of High Resolution NMR in Solids (SpringerVerlag, Berlin, 1983).

${ }^{17}$ J. H. Van Vleck, Phys. Rev. 33, 467 (1929).

${ }^{18}$ M. Goldman, P. J. Grandinetti, A. Llor, Z. Olegniczak, J. R. Sachleben, and J. W. Zwanzigger, J. Chem. Phys. 97, 8947 (1992).

${ }^{19}$ M. Wagner, Unitary Transformations in Solid State Physics (North Holland, Amsterdam, 1986).

${ }^{20}$ M. Goldman, J. Magn. Reson. 60, 437 (1984). 